\title{
Evaluation of the atrophogenic potential of different glucocorticoids using optical coherence tomography, 20-MHz ultrasound and profilometry; a double-blind, placebo-controlled trial
}

\author{
M. Coßmann and J. Welzel* \\ Department of Dermatology, University of Lübeck, Lübeck, Germany \\ *Department of Dermatology, General Hospital Augsburg, Stenglinstraße 2, 86156 Augsburg, Germany
}

\section{Summary}

\section{Correspondence \\ Julia Welzel. \\ E-mail: julia.welzel@klinikum-augsburg.de \\ Accepted for publication \\ 9 February 2006}

\section{Key words}

epidermal thickness, glucocorticoids, optical coherence tomography, profilometry, randomized controlled trial, skin atrophy, ultrasound

\section{Conflicts of interest}

None declared.
Background Skin atrophy is one of the main side-effects of topical corticosteroid therapy. Although the use of high-frequency ultrasound is an established method that has been studied previously, it allows measurements of the slow-reacting dermal thickness only.

Objectives To investigate the decreasing epidermal thickness, which occurs earlier, we used optical coherence tomography (OCT), a high-resolution noninvasive imaging technique, and compared it with 20-MHz ultrasound and profilometry.

Patients/methods In this double-blind placebo-controlled trial 20 healthy volunteers applied four different corticosteroids and the cream base formulation as placebo to the volar part of both arms once a day over a 4 -week period. The epidermal thickness, the dermal thickness and the skin surface roughness were assessed using OCT, high-frequency ultrasound and profilometry.

Results Each of the three methods allowed the detection and monitoring of significant corticosteroid-induced skin atrophy and its reversibility. The changes correlated with the potency of the steroids. The epidermal thickness decreased significantly in all test areas, even in the placebo and the untreated fields. As expected, the reduction in epidermal thickness was more pronounced and could be detected earlier by OCT than the reduction of dermal thickness using ultrasound. The epidermal surface roughness investigated using profilometry showed a slight smoothing.

Conclusions OCT allows a simple, fast and noninvasive in vivo measurement of the epidermal thickness. To evaluate the atrophogenic potential of corticosteroids it is more suitable than high-frequency ultrasound as epidermal thickness decreases earlier. In addition, epidermal thickness is a more sensitive indicator of steroid atrophy as the degree of thinning is much higher compared with the dermal atrophy. Profilometry might give further information; however, it would not be suitable for clinical use as the results were generally less pronounced. In the future, OCT might be useful to detect corticosteroid-induced side-effects at the beginning for monitoring the therapy.
Glucocorticoids have been used topically in dermatology since 1952. ${ }^{1}$ Today they represent one of the most important treatments in dermatological therapy. However, systemic and local side-effects are feared complications of their use. The most relevant adverse effect in dermatology is skin atrophy, depending on the duration and the method of application as much as the chemical structure and the potency of the steroid. ${ }^{2,3}$
In clinical and experimental studies high-frequency ultrasound has been used as an established noninvasive method for the quantification of corticosteroid-induced skin atrophy. ${ }^{4-11}$ However, because of its low resolution ultrasound determines only the dermal thickness. This parameter changes relatively late because of the slow metabolism of the collagen synthesis. Epidermal thickness decreases considerably earlier during 
corticosteroid treatment because of the high proliferation rate of the keratinocytes. ${ }^{3,12-14}$

Optical coherence tomography (OCT) is a noninvasive high-resolution method, which enables the evaluation of epidermal thickness. ${ }^{15-18}$ It would be useful to detect and monitor early side-effects such as skin atrophy.

In the double-blind trial, we used OCT in comparison with 20-MHz ultrasound and profilometry to investigate the atrophogenic potential of four different topical glucocorticoids and placebo during a 4 -week treatment in 20 healthy volunteers. The reversibility of the atrophic effect was examined 3 weeks after the end of treatment.

\section{Patients and methods}

\section{Optical coherence tomography}

The principle of OCT is based on interferometry. Light is coupled into optical fibres and divided into a reference beam with a known path length and a probe beam that is focused on the skin. The light in the probe beam is backscattered from the superficial skin layers and recombined again with the reference beam. Interference occurs only if the path lengths of both beams match to within the coherence length of the light source. The interference signal provides information on the path length distribution of the probe beam because of optical inhomogeneities of the tissue. By lateral scanning, OCT provides two-dimensional cross-sectional images of the skin in real time without touching the surface of the skin.

In this study we used a prototype of the OCT system Sirius (4optics, Lübeck, Germany) with an axial and lateral resolution of about $15 \mu \mathrm{m}$. The superluminescence diode (Superlum, Moscow, Russia) has a coherence length of $15 \mu \mathrm{m}$ and a wavelength of $1300 \mathrm{~nm}$. The two-dimensional images have a lateral dimension of 4-6 $\mathrm{mm}$ and a detection depth of about $1.5 \mathrm{~mm}$. A single-mode fibreoptic serves as an interferometer. A flexible hand piece allows measurements of any skin region. In an area of interest, an average A-scan representing the depth-resolved signal intensities in the probe can be calculated. On the curve, distances between peaks, signal intensities and the signal attenuation coefficient $(\mu)$ as a function of the slope of the curve can be calculated at different depths. The epidermal thickness can be determined by calculating the mean difference between the entrance peak of the A-scan signal representing the surface and the increase to the second peak at the papillary dermis. ${ }^{16-19}$

\section{High-frequency ultrasound}

We followed the established method of investigating corticosteroid-induced skin atrophy by using a 20-MHz ultrasound system (DermaScan $C^{\circledR}$ Ver. 3; Cortex Technology, Hadsund, Denmark) to assess the dermal thickness. The transducer is coupled to the skin by a water path and an ultrasonic gel film. The detection depth is about $1 \mathrm{~cm}$, the axial resolution about $80 \mu \mathrm{m}$ and the lateral resolution about $200 \mu \mathrm{m}$. Because of

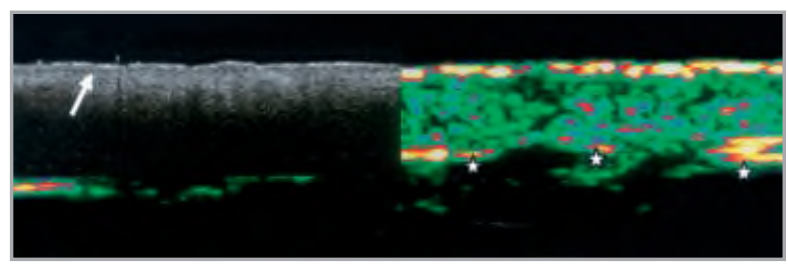

Fig 1. Optical coherence tomography (OCT) image of healthy skin (volar arm) projected true to scale on to the corresponding $20-\mathrm{MHz}$ ultrasound image of the same area. In the OCT image the first layer is the epidermis. The dermal-epidermal junction is marked by an arrow. In the ultrasound image it is not possible to delimit the epidermis from the dermis, whereas the border to the subcutis is clearly visible because of the higher detection depth (stars).

this low resolution it is not possible to distinguish the epidermal from the dermal layer so that dermal thickness always means epidermis plus dermis (Fig. 1). The dermal thickness was measured by calculating the mean distance between a line imputed just below the entrance signal and a second line drawn manually at the border between the echodense dermis and the echopoor subcutaneous fat. The number and intensity of reflections in the dermis can be quantified as echodensity.

\section{Profilometry}

The epidermal surface structure was investigated using optical three-dimensional profilometry (PRIMOS 4.0; GFMesstechnik $\mathrm{GmbH}$, Teltow, Germany). It is a noninvasive in vivo method to measure the skin roughness within a few seconds and without need of any replicas. A camera image and a depth-resolved surface profile by fringe projection were collected simultaneously in vivo from the test sites. The image size was $14 \times 18 \mathrm{~mm}$. The profiles were adjusted to a polynome of 5 degrees of freedom into both axes to remove the arch of the arm. The roughness parameters Ra (arithmetic mean of roughness) and Rz (mean roughness) were calculated after filtering (wave filter according to DIN 4768) along 16 star-shaped lines of the profile.

\section{Volunteers}

The treatments and measurements in this double-blind trial were performed on healthy skin of 20 volunteers (15 women and five men; age range 22-56 years). Exclusion criteria were any systemic or topical glucocorticoid treatment within 2 months before and any skin disease or allergic skin reaction in the past. Before enrolment, each subject was fully informed about the ingredients and possible side-effects of the preparations and gave signed and informed consent. The experimental protocol was approved by the ethics committee of the University of Lübeck (reference 02-083).

\section{Treatments}

The volunteers applied five different treatments to the volar part of both arms once daily over a period of 4 weeks. The treatments were: 
1 Hydrocortisone 1\%; potency class I, mild.

2 Methylprednisolone aceponate $0 \cdot 1 \%$; new class with high potency and low risk of side-effects.

3 Betamethasone valerate $0 \cdot 1 \%$; potency class III, potent.

4 Clobetasol propionate $0.05 \%$; potency class IV, very potent.

5 Cream base, steroid free.

One adjacent test area without any treatment served as control. The different ointments were randomly assigned to the six test areas $(5 \times 5 \mathrm{~cm}$ each) of each person. They were numbered and rotated from subject to subject. For application, the volunteers were encouraged to use another finger for each preparation, not to cover the treated areas within the next 10 min and not to wash them during the following $2 \mathrm{~h}$. The volunteers were advised to apply an amount of about a grain, equivalent to about $50 \mathrm{mg}$, to each area.

\section{Measurements}

Measurements were taken on days 0 (before treatment), 3, 7, $14,21,28$ and 3 weeks after the end of the treatment on day 49 using OCT, 20-MHz ultrasound and profilometry. Because of a possible influence of the ultrasonic gel the ultrasound measurements were always performed after OCT and profilometry. $^{20}$

\section{Statistical analysis}

The Wilcoxon signed rank test for matched pairs was used for comparison of the values before and during the treatment.

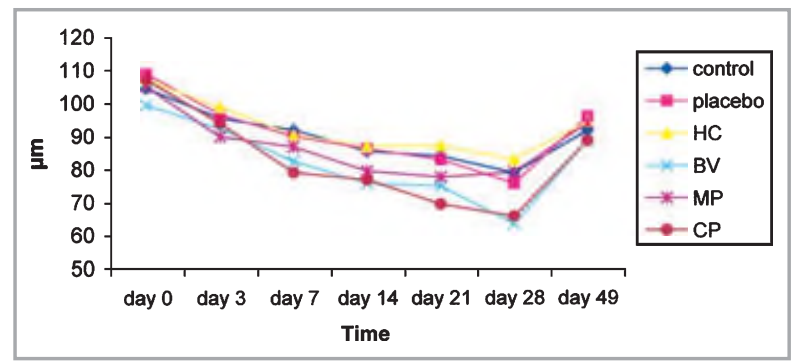

Fig 2. Mean epidermal thickness $(\mu \mathrm{m})$ measured by optical coherence tomography (OCT). HC, hydrocortisone; BV, betamethasone valerate; MP, methylprednisolone aceponate; $\mathrm{CP}$, clobetasol propionate.
Statistical evaluation of differences between the treatments was performed using the Friedman variance analysis of ranks with consecutive Wilcoxon/Wilcox comparisons. Significance of differences was assumed at $\mathrm{P} \leq 0 \cdot 05$.

\section{Results}

\section{Optical coherence tomography}

The epidermal thickness decreased continuously in all test areas, even in the placebo and the untreated area (Fig. 2). After 7 days of treatment at the latest, this thinning was significant $(P \leq 0.01)$ for all test fields (Table 1). Three weeks after the end of treatment (day 49) epidermal thickness had reached almost its baseline value, but was still significantly different from the thickness before treatment in all areas. The increase between days 28 and 49 was significant in all fields except the methylprednisolone aceponate treatment.

The degree of epidermal thinning reflects the different potency of the treatments: clobetasol propionate induced the highest degree of atrophy (Figs 3, 4), followed by betamethasone valerate and methylprednisolone aceponate. Hydrocortisone, placebo and the control induced atrophy to a lower, but still significant, extent.

The signal attenuation coefficient $(\mu)$ representing the scattering of the upper dermis showed a slight but significant increase in all test fields. There was no significant difference between the preparations.

\section{Ultrasound}

The dermal thickness measured by $20-\mathrm{MHz}$ ultrasound showed a decrease in all fields, which was generally less pronounced than the decrease in epidermal thickness evaluated by OCT (Fig. 5). Unlike OCT, the difference between corticosteroid treatment and placebo or control is now statistically visible: dermal thinning of steroid-treated skin was significant after 3 days, that of placebo and untreated skin not until 21 days (Table 2). Three weeks after the end of application dermal thickness had regained almost its initial value. This increase was significant in all test areas except the placebo and the hydrocortisone-treated areas.

Table 1 Optical coherence tomography results of the epidermal thickness

\begin{tabular}{lccccccc}
\hline & Day 0 (T0) & \multicolumn{1}{c}{ Day 3 (T1) } & Day 7 (T2) & Day 14 (T3) & Day 21 (T4) & Day 28 (T5) & Day 49 (T6) \\
\hline Control & $104 \cdot 7 \pm 28 \cdot 2$ & $95 \cdot 6 \pm 20 \cdot 6 *$ & $92 \cdot 0 \pm 20 \cdot 7$ & $85 \cdot 5 \pm 21 \cdot 4$ & $84 \cdot 1 \pm 25 \cdot 2$ & $79 \cdot 0 \pm 22 \cdot 7$ & $91 \cdot 8 \pm 22 \cdot 1 *$ \\
Placebo & $109 \cdot 2 \pm 18 \cdot 0$ & $96 \cdot 8 \pm 18 \cdot 5 * *$ & $90 \cdot 1 \pm 17 \cdot 9$ & $86 \cdot 3 \pm 18 \cdot 3$ & $83 \cdot 1 \pm 22 \cdot 0$ & $75 \cdot 8 \pm 21 \cdot 5$ & $96 \cdot 1 \pm 22 \cdot 0 *$ \\
HC & $107 \cdot 2 \pm 25 \cdot 6$ & $99 \cdot 1 \pm 20 \cdot 0 *$ & $90 \cdot 4 \pm 16 \cdot 1$ & $87 \cdot 1 \pm 15 \cdot 0$ & $87 \cdot 3 \pm 20 \cdot 7$ & $83 \cdot 1 \pm 23 \cdot 5$ & $94 \cdot 6 \pm 17 \cdot 1 *$ \\
BV & $99 \cdot 6 \pm 21 \cdot 6$ & $92 \cdot 4 \pm 13 \cdot 7$ & $82 \cdot 6 \pm 17 \cdot 6 * *$ & $75 \cdot 9 \pm 19 \cdot 9$ & $75 \cdot 1 \pm 15 \cdot 8$ & $63 \cdot 5 \pm 13 \cdot 4$ & $88 \cdot 8 \pm 20 \cdot 1 * *$ \\
MP & $105 \cdot 2 \pm 21 \cdot 3$ & $90 \cdot 0 \pm 22 \cdot 4 * *$ & $86 \cdot 9 \pm 21 \cdot 9$ & $79 \cdot 6 \pm 23 \cdot 0$ & $77 \cdot 8 \pm 27 \cdot 1$ & $79 \cdot 1 \pm 31 \cdot 5$ & $94 \cdot 1 \pm 22 \cdot 7$ \\
CP & $107 \cdot 4 \pm 15 \cdot 9$ & $94 \cdot 1 \pm 28 \cdot 3 * *$ & $79 \cdot 1 \pm 18 \cdot 1$ & $77 \cdot 0 \pm 25 \cdot 0$ & $69 \cdot 5 \pm 18 \cdot 3$ & $65 \cdot 8 \pm 19 \cdot 7$ & $88 \cdot 8 \pm 17 \cdot 2 * *$ \\
\hline
\end{tabular}

Mean $\pm \mathrm{SD}, \mathrm{n}=20, * \mathrm{P} \leq 0.05, * * \mathrm{P} \leq 0.01$; the asterisks mark the first significant change compared with $\mathrm{T} 0$ of the same area, at T6 compared with T5. HC, hydrocortisone; BV, betamethasone valerate; MP, methylprednisolone aceponate; $\mathrm{CP}$, clobetasol propionate 


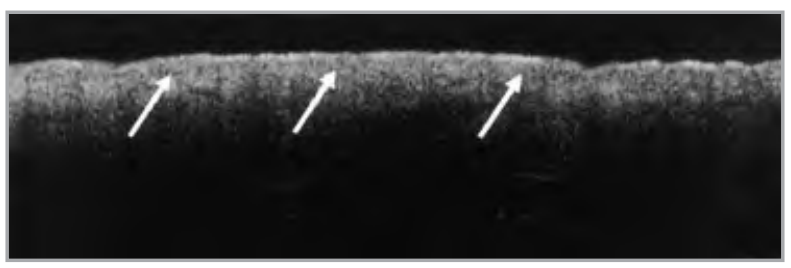

Fig 3. Optical coherence tomography image of healthy skin at the volar part of the arm before treatment with clobetasol propionate (day 0 , subject no. 16). Arrows mark the dermal-epidermal junction. Image area $6 \times 1.94 \mathrm{~mm}$.

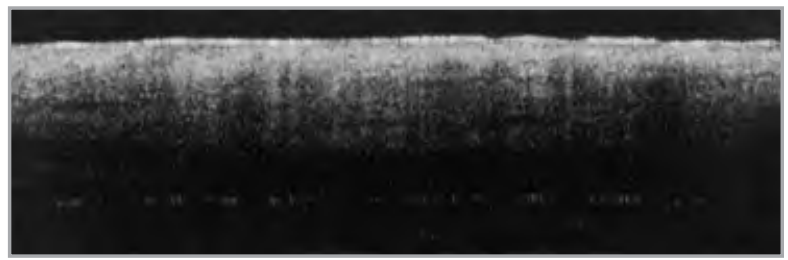

Fig 4. Optical coherence tomography image of the same area after 4 weeks of treatment with clobetasol propionate (day 28, subject no. 16). The epidermal thickness decreased markedly. Image area $6 \times 1.94 \mathrm{~mm}$.

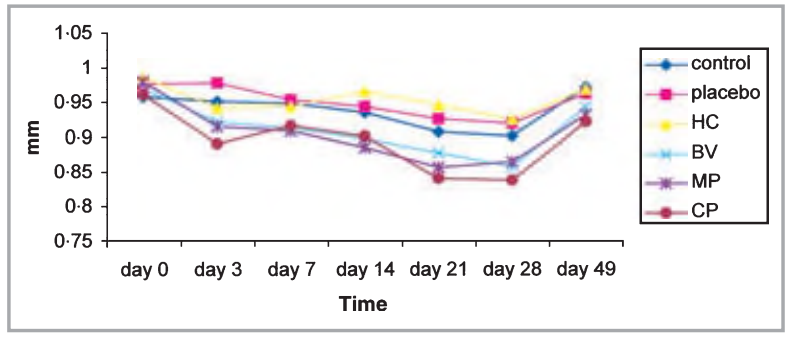

Fig 5. Mean dermal thickness (mm) measured by $20-\mathrm{MHz}$ ultrasound. HC, hydrocortisone; BV, betamethasone valerate; MP, methylprednisolone aceponate; $\mathrm{CP}$, clobetasol propionate.

The ultrasound results represent, like OCT, the different potency of the preparations: dermal thinning because of clobetasol propionate is statistically different from that caused by hydrocortisone and placebo (Figs 6, 7).

The echodensity increase was statistically significant after 7 days at the latest in all areas. The differences between the treatments were less pronounced compared with dermal thickness.

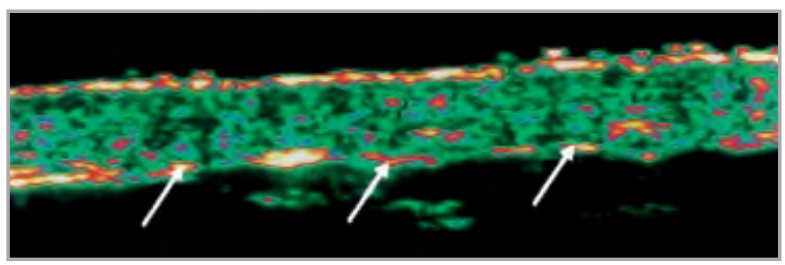

Fig 6. Ultrasound (20-MHz) image of healthy skin at the volar part of the arm before treatment with clobetasol propionate (day 0, subject no. 16). Arrows mark the border between dermis and subcutis. Image area $12 \times 3.9 \mathrm{~mm}$.

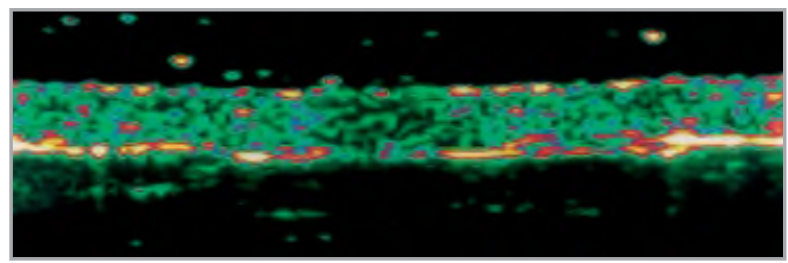

Fig 7. Ultrasound (20-MHz) image of the same area after 4 weeks of treatment with clobetasol propionate (day 28 , subject no. 16). The dermal thinning is visible. Image area $12 \times 3.9 \mathrm{~mm}$.

\section{Profilometry}

The epidermal surface roughness evaluated by profilometry showed a slight reduction represented by slightly decreasing $\mathrm{Rz}$ and $\mathrm{Ra}$ values, especially in the clobetasol propionate-treated area (Figs 8-10). After 7 days, this decrease was significant in the test fields of clobetasol propionate, betamethasone valerate and methylprednisolone aceponate, and after 21 days in

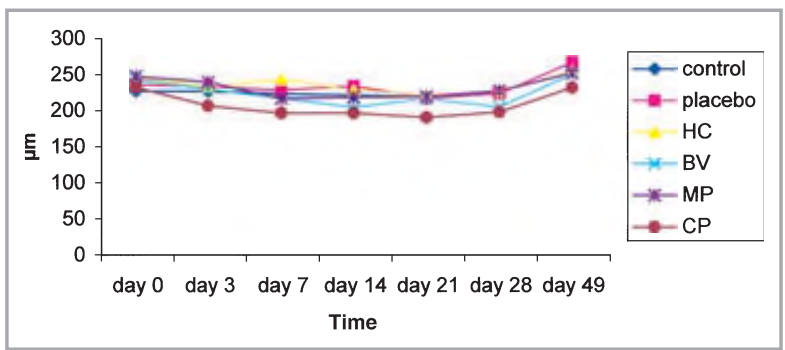

Fig 8. Mean roughness Rz ( $\mu \mathrm{m})$ measured by profilometry. HC, hydrocortisone; BV, betamethasone valerate; MP, methylprednisolone aceponate; $\mathrm{CP}$, clobetasol propionate.

Table 2 Ultrasound results of the dermal thickness

\begin{tabular}{|c|c|c|c|c|c|c|c|}
\hline & Day 0 (T0) & Day 3 (T1) & Day 7 (T2) & Day 14 (T3) & Day 21 (T4) & Day 28 (T5) & Day 49 (T6) \\
\hline Control & $0.961 \pm 0.14$ & $0.954 \pm 0.16$ & $0.951 \pm 0.16$ & $0.938 \pm 0.15$ & $0 \cdot 911 \pm 0.15^{* *}$ & $0.905 \pm 0.13$ & $0.974 \pm 0.14 *$ \\
\hline Placebo & $0.980 \pm 0.12$ & $0.981 \pm 0.13$ & $0.957 \pm 0.14$ & $0.947 \pm 0.16$ & $0.929 \pm 0.17 * *$ & $0.923 \pm 0.15$ & $0.965 \pm 0.12$ \\
\hline $\mathrm{HC}$ & $0.990 \pm 0.13$ & $0.945 \pm 0.12 *$ & $0.946 \pm 0.12$ & $0.967 \pm 0.16$ & $0 \cdot 949 \pm 0 \cdot 17$ & $0.928 \pm 0.14$ & $0.971 \pm 0.15$ \\
\hline BV & $0.973 \pm 0.15$ & $0.925 \pm 0.16^{*}$ & $0.917 \pm 0.14$ & $0.901 \pm 0.16$ & $0.881 \pm 0.16$ & $0.860 \pm 0.18$ & $0.946 \pm 0.13 * *$ \\
\hline MP & $0.983 \pm 0.15$ & $0.918 \pm 0.12^{* *}$ & $0 \cdot 913 \pm 0 \cdot 14$ & $0.889 \pm 0.13$ & $0.860 \pm 0.13$ & $0.868 \pm 0.16$ & $0.935 \pm 0.11 * *$ \\
\hline $\mathrm{CP}$ & $0.964 \pm 0.14$ & $0.894 \pm 0.14^{* *}$ & $0.920 \pm 0.18$ & $0.905 \pm 0.17$ & $0.843 \pm 0.17$ & $0.841 \pm 0.16$ & $0.925 \pm 1.17 *$ \\
\hline
\end{tabular}

Mean $\pm \mathrm{SD}, \mathrm{n}=20, * \mathrm{P} \leq 0 \cdot 05, * * \mathrm{P} \leq 0 \cdot 01$; the asterisks mark the first significant change compared with T0 of the same area, at T6 compared with T5. HC, hydrocortisone; BV, betamethasone valerate; MP, methylprednisolone aceponate; $\mathrm{CP}$, clobetasol propionate 


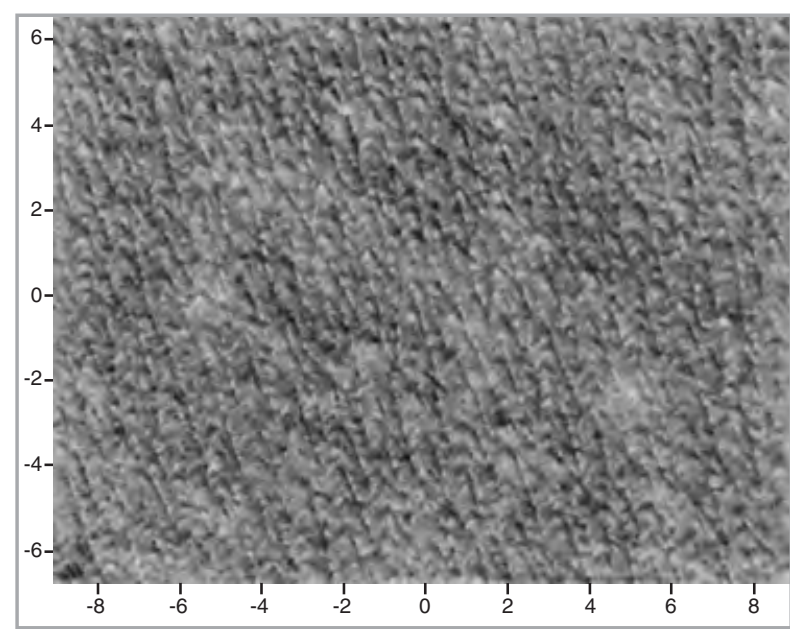

Fig 9. Profilometry image of healthy skin at the volar part of the arm before treatment with clobetasol propionate (day 0, subject no. 16). Image area $12 \times 16 \mathrm{~mm}$.

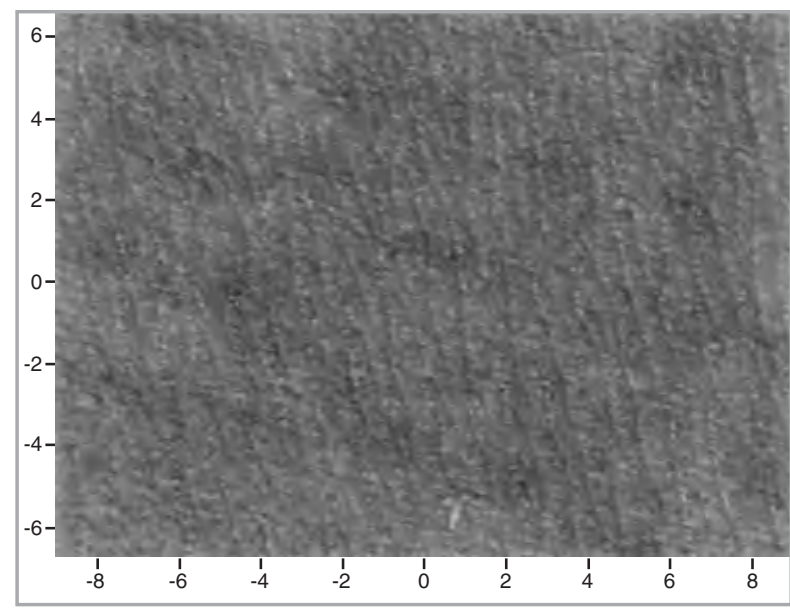

Fig 10. Profilometry image of the same area after 4 weeks of treatment with clobetasol propionate (day 28, subject no. 16). The surface roughness is visibly reduced. Image area $12 \times 16 \mathrm{~mm}$.

the hydrocortisone-treated area (Table 3). The placebo and the control areas showed no significant reduction in skin roughness, even after 4 weeks. The increase 3 weeks after the end of treatment was statistically significant in all test fields.
The different extent of decreasing roughness between clobetasol propionate and hydrocortisone or placebo was significant only on day 7 .

\section{Comparison of the methods}

Each of the three methods allowed the detection and monitoring of corticosteroid-induced skin atrophy. As expected, the reduction of epidermal thickness compared with the dermal thickness was more pronounced and included even the placebo and the untreated area. The epidermal atrophy could be detected earlier by OCT than the reduction of dermal thickness assessed by ultrasound. However, the epidermal roughness evaluated by profilometry showed only slight changes, which were significant for the potent corticosteroids. The signal attenuation coefficient $(\mu)$ as well as the echodensity of the upper dermis showed a slight but significant increase.

Furthermore, the reversibility of the atrophogenic effect within 3 weeks after the end of treatment could be demonstrated as statistically significant by all the methods. At the end, only the epidermal thickness was still significantly different from its baseline values.

The results of OCT, ultrasound and profilometry correlated with the potency of the different steroids. Clobetasol propionate induced the strongest atrophogenic effect, followed by betamethasone valerate and methylprednisolone aceponate. The difference between hydrocortisone-induced atrophy and that seen in placebo or untreated skin was not statistically significant.

Figure 11 demonstrates the differences between the methods: the changes in epidermal thickness, dermal thickness and epidermal surface roughness assessed by OCT, ultrasound and profilometry, respectively, are clearly shown for clobetasol propionate, the treatment with the most atrophogenic effect. The epidermal thickness measured by OCT clearly showed the highest degree of change in comparison with ultrasound and profilometry.

\section{Discussion}

OCT allowed the quantification of corticosteroid-induced skin atrophy. Moreover, the epidermal thickness measured by this new method was obviously a more sensitive parameter than

Table 3 Profilometry results of the mean roughness (Rz)

\begin{tabular}{|c|c|c|c|c|c|c|c|}
\hline & Day 0 (T0) & Day 3 (T1) & Day 7 (T2) & Day 14 (T3) & Day 21 (T4) & Day 28 (T5) & Day 49 (T6) \\
\hline Control & $228 \cdot 0 \pm 54 \cdot 8$ & $228 \cdot 1 \pm 41 \cdot 1$ & $224 \cdot 4 \pm 43 \cdot 1$ & $222 \cdot 1 \pm 28 \cdot 4$ & $220 \cdot 1 \pm 40 \cdot 5$ & $227 \cdot 7 \pm 37 \cdot 5$ & $255.6 \pm 37.3 *$ \\
\hline Placebo & $237 \cdot 8 \pm 41 \cdot 0$ & $234 \cdot 3 \pm 39 \cdot 9$ & $229 \cdot 7 \pm 39 \cdot 4$ & $235 \cdot 0 \pm 47 \cdot 2$ & $218 \cdot 3 \pm 36 \cdot 8$ & $224 \cdot 6 \pm 36 \cdot 2$ & $268 \cdot 0 \pm 62 \cdot 4^{*}$ \\
\hline $\mathrm{HC}$ & $246 \cdot 8 \pm 49 \cdot 7$ & $234 \cdot 0 \pm 53 \cdot 7$ & $244 \cdot 6 \pm 64 \cdot 1$ & $229 \cdot 3 \pm 28.9$ & $223 \cdot 1 \pm 30 \cdot 2 *$ & $227 \cdot 6 \pm 43 \cdot 5$ & $255.7 \pm 53.3 * *$ \\
\hline BV & $243 \cdot 9 \pm 43 \cdot 6$ & $230 \cdot 9 \pm 45 \cdot 9$ & $218 \cdot 1 \pm 26 \cdot 0 * *$ & $205 \cdot 7 \pm 43 \cdot 5$ & $217 \cdot 4 \pm 32 \cdot 8$ & $206 \cdot 3 \pm 45 \cdot 8$ & $251 \cdot 2 \pm 72 \cdot 4 * *$ \\
\hline MP & $248.9 \pm 44.6$ & $240.8 \pm 58.9$ & $217 \cdot 9 \pm 47 \cdot 1 * *$ & $219 \cdot 8 \pm 56 \cdot 7$ & $220 \cdot 9 \pm 41 \cdot 2$ & $228 \cdot 7 \pm 57 \cdot 2$ & $253.5 \pm 53.1 *$ \\
\hline $\mathrm{CP}$ & $233 \cdot 4 \pm 55 \cdot 2$ & $207 \cdot 9 \pm 40 \cdot 0$ & $197 \cdot 7 \pm 34 \cdot 5 *$ & $197 \cdot 7 \pm 36 \cdot 4$ & $191 \cdot 7 \pm 45 \cdot 7$ & $199 \cdot 3 \pm 38 \cdot 4$ & $233.0 \pm 55.5 *$ \\
\hline
\end{tabular}

Mean $\pm \mathrm{SD}, \mathrm{n}=20, * \mathrm{P} \leq 0.05, * * \mathrm{P} \leq 0.01$; the asterisks mark the first significant change compared with T0 of the same area, at $\mathrm{T} 6$ compared with T5. HC, hydrocortisone; BV, betamethasone valerate; MP, methylprednisolone aceponate; CP, clobetasol propionate 


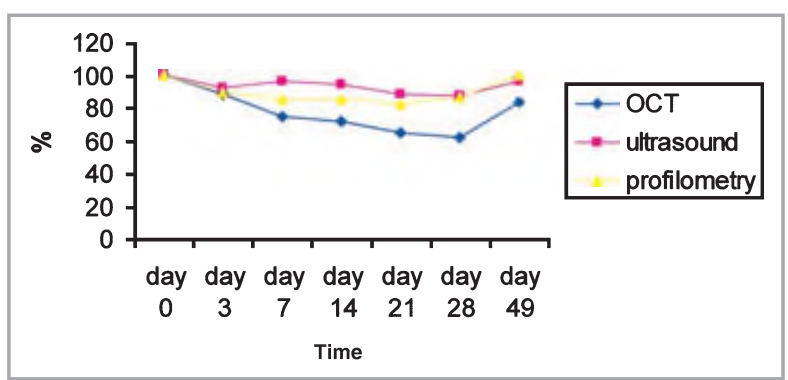

Fig 11. Changes (in percentage) of the epidermal thickness [optical coherence tomography (OCT)], the dermal thickness (ultrasound) and the mean roughness (profilometry) in the clobetasol propionatetreated skin of all subjects.

dermal thickness assessed by ultrasound: the decrease in epidermal thickness started earlier and was more pronounced than the reduction in dermal thickness. This corresponds to the natural mechanism of steroid atrophy: the epidermis, with the high proliferation rate of keratinocytes, is affected earlier than the dermis, which changes relatively late because of the low production rate of collagen and mucopolysaccharides based on a changed m-RNA synthesis. ${ }^{3,12-14}$ Hence, OCT is a more suitable method for the assessment of corticosteroid-induced skin atrophy than high-frequency ultrasound-until now the established noninvasive method for these investigations.

It is remarkable that OCT detected a significant decrease even in the placebo and the untreated area after 3 days whereas this effect was not detectable at all, as early and to the same extent, by ultrasound and profilometry. This might be because of a slight systemic effect, which was first noticeable in the epidermal thickness. Another reason might be a spreading of the creams from adjacent areas. But the decoding of the random distribution of the test fields on the arms at the end of the trial revealed that the degree of epidermal thinning in the untreated area was completely independent of the potency of the adjacent treatment. From this irregularity an unexpected slight systemic effect on the whole skin can be postulated, more or less pronounced from subject to subject. Of course this is a dose-dependent effect.

In 1999, Pagnoni et al. ${ }^{21}$ investigated corticosteroid-induced epidermal thinning using OCT, but under the treatment of only clobetasol propionate for 3 weeks. At the end of this trial, the epidermal thickness in the test field was reduced by 17.6\%; no systemic effect has been described. In our study, a reduction of epidermal thickness by $35 \cdot 3 \%$ could be observed. A systemic effect-more likely to appear under the treatment of four different corticosteroids at both arms in a 4-week period, in contrast to only one corticosteroid in a 3 -week period-might explain these different results. It is obvious that a possible systemic effect would be first detectable by assessing the early-reacting epidermis, which underlines the importance of OCT in clinical and therapeutic use: local and systemic side-effects of a corticosteroid therapy may be detected and monitored earlier than was previously possible by using OCT instead of the established methods, i.e. ultrasound.
A further advantage of OCT is that the epidermal thickness is not or is hardly influenced by physiological factors in contrast to the dermal thickness, which is influenced, for example, by age, time of day, body water distribution, ultraviolet exposure or sex hormones. ${ }^{22-24}$ The epidermal surface roughness is also influenced by different parameters: it increases, for example, in a dry environment or at a low skin hydration. ${ }^{25-27}$ The epidermal thickness is less influenced by those factors; optical properties such as the light attenuation coefficient might be more affected. ${ }^{20}$ Therefore, OCT measurements show less variability compared with dermal thickness and skin surface roughness assessed by ultrasound and profilometry.

Looking at the reversibility of the atrophogenic effect it is remarkable that the epidermal thickness has not reached its baseline values. The thickness results of OCT on day 49 are still statistically different from day 0 in all test areas, whereas the dermal thickness results of ultrasound differ significantly from baseline values only in the clobetasol propionate- and methylprednisolone aceponate-treated areas. Regeneration of the normal proliferation rate of the strongly influenced epidermal keratinocytes seems to take longer than the first effect of dermal regeneration: the re-activation of the just slightly suppressed glycosaminoglycane synthesis with consecutive rehydration and thickening of the dermis. Hence, steroid atrophy of the epidermis can be characterized by an earlier beginning and a longer duration compared with the dermis.

Because of the antiproliferative effect of corticosteroids, a decrease in skin surface roughness was expected and confirmed by the profilometry results. In spite of these conclusive results, in contrast to further investigations using profilometry, ${ }^{7,28,29}$ the steroid effect was generally less pronounced compared with OCT and ultrasound. Profilometry gives further information about steroid atrophy; however, it would not be suitable for monitoring corticosteroid effects in clinical use.

The differences between the potency of the corticosteroids was demonstrated by each of the methods. Methylprednisolone aceponate, a new-generation steroid with an increased risk-benefit ratio, actually induced less atrophy than the potent steroids (class III and IV). However, a very early significant reduction, not only in epidermal thickness, which could be well-founded on the above-mentioned systemic effect, but also in dermal thickness, could be observed. This is remarkable considering the fact that clinical trials with topical methylprednisolone aceponate treatment observed no steroid atrophy at all after a period of 12 weeks. Hence, the atrophogenic potential of those new corticosteroids should be re-investigated.

In summary, OCT allows a simple, fast and noninvasive evaluation of the atrophogenic potential of topical corticosteroids. It is more suitable than high-frequency ultrasound as epidermal thickness decreases earlier than dermal thickness. Epidermal thickness is also a more sensitive indicator of steroid atrophy as OCT allowed the detection of a possible systemic effect, in contrast to ultrasound results. Profilometry might give further information, but the effects were less pronounced. The results correlated with the potency of the 
steroids, although methylprednisolone aceponate induced an unexpected early atrophy.

In clinical use, OCT might be of value not only for the monitoring of corticosteroid therapy but also for choosing the optimal treatment for inflammatory skin disease as it enables the detection of side-effects at the beginning of treatment.

\section{References}

1 Sulzberger MB, Witten VH. The effect of topically applied compound F in selected dermatoses. J Invest Dermatol 1952; 19:101-2.

2 Sneddon IB. Atrophy of the skin. The clinical problems. Br J Dermatol 1976; 94:121-3.

3 Mills CM, Marks R. Side effects of topical glucocorticoids. Curr Probl Dermatol 1993; 21:122-31.

4 Kerscher MJ, Korting HC. Topical glucocorticoids of the non-fluorinated double-ester type. Lack of atrophogenicity in normal skin as assessed by high-frequency ultrasound. Acta Derm Venereol 1992; 72:214-16.

5 Kolbe L, Kligman AM, Schreiner V et al. Corticosteroid-induced atrophy and barrier impairment measured by non-invasive methods in human skin. Skin Res Technol 2001; 7:73-7.

6 Korting HC. Topical glucocorticoids and thinning of normal skin as to be assessed by ultrasound. Curr Probl Dermatol 1993; 21:114-21.

7 Korting HC, Unholzer A, Schäfer-Korting M et al. Different skin thinning potential of equipotent medium-strength glucocorticoids. Skin Pharmacol Appl Skin Physiol 2002; 15:85-91.

8 Hoffmann K, Auer T, Stücker M et al. Comparison of skin atrophy and vasoconstriction due to mometasone furoate, methylprednisolone and hydrocortisone. J Eur Acad Dermatol Venereol 1998; 10:13742.

9 Levy J, Gassmuller J, Schroder G et al. Comparison of the effects of calcipotriol, prednicarbate and clobetasol 17-propionate on normal skin assessed by ultrasound measurement of skin thickness. Skin Pharmacol 1994; 7:231-6.

10 Tan CY, Statham B, Marks R et al. Skin measurement by pulsed ultrasound: its reproducibility, validation and variability. Br J Dermatol 1982; 106:657-67.

11 Kerscher MJ, Hart H, Korting $\mathrm{HC}$ et al. In vivo assessment of the atrophogenic potency of mometasone furoate, a newly developed chlorinated potent topical glucocorticoid as compared to other topical glucocorticoids old and new. Int J Clin Pharmacol Ther 1995; 33:187-9.

12 Goa KL. Clinical pharmacology and pharmacokinetic properties of topically applied corticosteroids: a review. Drugs 1988; 36:51-61.

13 Oikarinen A, Haapasaari KM, Sutinen M et al. The molecular basis of glucocorticoid-induced skin atrophy: topical glucocorticoid decreases both collagen synthesis and the corresponding collagen
mRNA level in human skin in vivo. Br J Dermatol 1998; 139:110610.

14 Wilson-Jones E. Steroid atrophy-a histological appraisal. Dermatologica 1976 ; 152:107-15.

15 Gladkova ND, Petrova A, Nikulin NK et al. In vivo optical coherence tomography imaging of the skin: norm and pathology. Skin Res Technol 2000; 6:6-16.

16 Welzel J. Optical coherence tomography: a review. Skin Res Technol 2001; 7:1-9.

17 Welzel J, Lankenau E, Birngruber R et al. Optical coherence tomography of the human skin. J Am Acad Dermatol 1997; 37:958-63.

18 Pan Y, Lankenau E, Welzel J et al. Optical coherence-gated imaging of biological tissues. IEEE J Selected Topics Quant Elect 1996; 2:102934.

19 Fujimoto JG, Pitris C, Boppart SA et al. Optical coherence tomography: an emerging technology for biomedical imaging and optical biopsy. Neoplasia 2000; 2:9-25.

20 Welzel J, Reinhardt C, Lankenau E et al. Changes in function and morphology of normal human skin: evaluation using optical coherence tomography. Br J Dermatol 2004; 150:220-5.

21 Pagnoni A, Knuettel A, Welker P et al. Optical coherence tomography in dermatology. Skin Res Technol 1999; 5:83-7.

22 Eisenbeiß C, Welzel J, Eichler W et al. Influence of body water distribution on skin thickness: measurements using high-frequency ultrasound. Br J Dermatol 2001; 144:947-51.

23 Eisenbeiß C, Welzel J, Schmeller W. The influence of female sex hormones on skin thickness: evaluation using $20 \mathrm{MHz}$ sonography. Br J Dermatol 1998; 139:462-7.

24 Gniadecka M, Serup J, Söndergaard J. Age-related diurnal changes of dermal oedema: evaluation by high-frequency ultrasound. Br J Dermatol 1994; 131:849-55.

25 Eberlein-Konig B, Schafer T, Huss-Marp J et al. Skin surface pH, stratum corneum hydration, transepidermal water loss and skin roughness related to atopic eczema and skin dryness in a population of primary school children. Acta Derm Venereol 2000; 80:18891.

26 Egawa M, Oguri M, Kuwahara T et al. Effect of exposure of human skin to a dry environment. Skin Res Technol 2002; 8:212-18.

27 Manuskiatti W, Schwindt DA, Maibach HI. Influence of age, anatomic site and race on skin surface roughness and scaliness. Dermatology 1998; 196:401-7.

28 Kerscher MJ, Korting HC, Mehringer L et al. 0.05\% clobetasol 17propionate cream and ointment but not the corresponding $0.1 \%$ triamcinolone acetonide preparations increase skin surface roughness: a possible dissociation of unwanted epidermal and dermal effects. Skin Pharmacol 1996; 9:120-3.

29 Wolff HH, Kreusch JF, Wilhelm KP et al. The psoriasis plaque test and topical corticosteroids: evaluation by computerised laser profilometry. Curr Probl Dermatol 1993; 21:107-13. 